\title{
Biquadratic exchange and critical behaviour in the diluted antiferromagnet $\mathrm{Eu}_{\mathrm{x}} \mathrm{Sr}_{1-\mathrm{x}} \mathrm{Te}$
}

\author{
U. Köbler ${ }^{1}$, I. Apfelstedt ${ }^{1, \star}$, K. Fischer ${ }^{1}$, W. Zinn ${ }^{1}$, E. Scheer ${ }^{2}$, J. Wosnitza ${ }^{2}$, H. v. Löhneysen ${ }^{2}$, T. Brückel ${ }^{3, \star \star}$ \\ ${ }^{1}$ Institut für Festkörperforschung, KFA Jülich, Postfach 1913, D-52425 Jülich, Germany \\ ${ }_{2}^{2}$ Physikalisches Institut, Universität Karlsruhe, Engesserstrasse 7, D-76131 Karlsruhe, Germany \\ ${ }^{3}$ Institut Laue-Langevin, F-38042 Grenoble, France
}

Received: 7 May 1993

\begin{abstract}
Magnetization, specific heat, magnetocaloric effect, and neutron diffraction measurements have been performed in order to better understand the magnetic interactions and phase transitions in the diluted antiferromagnetic system $\mathrm{Eu}_{\mathrm{x}} \mathrm{Sr}_{1-\mathrm{x}} \mathrm{Te}$. Due to the low Néel temperature of $T_{N}=9.8 \mathrm{~K}$ and the associated small critical field of $B_{C}(T=0)=7.5 \mathrm{~T}$, EuTe provides the opportunity to change the angle between the antiferromagnetic moments continuously between $180^{\circ}$ and $0^{\circ}$ by applying a magnetic field and allows thus to probe the type of the magnetic interaction. It is found that in addition to the common bilinear Heisenberg exchange mechanism there is evidence for a small biquadratic exchange term, which has important consequences on the critical behaviour of $\mathrm{Eu}_{x} \mathrm{Sr}_{1-x} \mathrm{Te}$. In the case of EuTe the biquadratic exchange supports the antiferromagnetic order, such that virtually no anisotropy exists and this restricts the antiferromagnetic (AF) to spin-flop (SF) transition to $B_{0}=0$. On dilution with strontium, the biquadratic interaction decreases strongly and changes its sign at $x \approx 0.83$ thus favouring the perpendicular moment orientation. This acts like an anisotropy on the dominating antiferromagnetic bilinear interaction and shifts the $\mathrm{AF}-\mathrm{SF}$ phase line to finite fields which reach values of $0.3 \mathrm{~T}$ for $T \rightarrow 0$. No bicritical behaviour is observed, but instead, the SF phase is the state of lowest energy in a small temperature interval below $T_{N}$. The critical exponent $\alpha(x)$ of the specific heat decreases in a non-linear way with dilution starting from a large positive value for EuTe. $\alpha(x)$ is close to the Heisenberg value -0.12 near $x \approx 0.83$ where the biquadratic interaction is zero. The same applies for the exponent $\beta$ of the sublattice magnetization.
\end{abstract}

PACS: 75.30.Et; 75.30.Kz; 75.40.-s

\footnotetext{
* Present address: Micro Parts, Griesbachstrasse 10, D-76185 Karlsruhe, Germany

$\star \star$ Present address: HASYLAB Notkestrasse 85, D-22607 Hamburg, Germany
}

\section{Introduction}

EuTe is a well known easy-plane antiferromagnet [1] having the same fcc-latice and magnetic structure as MnO. In the ordered state, the spin-only moments of the $\mathrm{Eu}^{2+}$ ions with $7 \mu_{B}$ are parallel to each other within (111) planes but alternate in direction between adjacent planes [2]. A small anisotropy within the (111) planes favours the [11 2 ] direction as the magnetic easy direction [3].

Until now the magnetic properties of EuTe have been described by $J_{1}$, the ferromagnetic exchange constant to the 12 nearest Eu neighbours and $J_{2}$, the antiferromagnetic exchange constant to the 6 next-nearest Eu neighbours. Spin-wave dispersion measurements on the ferromagnet EuS proved that the interactions to the third and more remote neighbours are in fact small there. While they are expected to increase towards EuTe in the Eumonochalogenide series their effect can be subsumed in the competing parameters $J_{1}$ and $J_{2}$ especially because of their different sign [4]. In a mean field model one can estimate these exchange parameters $J_{1}$ and $J_{2}$ from the experimental values of the Néel temperature $T_{N}$ and the paramagnetic ordering temperature $\Theta$ according to the well-known relations:

$$
\begin{aligned}
& k_{B} T_{N}=-\frac{2}{3} S(S+1) \cdot 6 J_{2} \\
& k_{B} \Theta=\frac{2}{3} S(S+1)\left[12 J_{1}+6 J_{2}\right] .
\end{aligned}
$$

It is important to note that the Néel temperature of EuTe is determined in the framework of this model by the nextnearest neighbour interaction $J_{2}$ alone since there are as many positive as negative spin orientations among the 12 nearest neighbours. This holds at least for the undiluted case $x=1$. Inserting $T_{N}=9.8 \mathrm{~K}$ and $\Theta=-3 \mathrm{~K}$ into (1) results in

$J_{1} / k_{B}=0.06 \mathrm{~K}$ and $J_{2} / k_{B}=-0.16 \mathrm{~K}$.

We should, however, note that the Heisenberg sum to the nearest and next-nearest neighbours allows no satisfac- 
tory description of all details of the observed magnetic properties of EuTe. This ansatz allows a consistent description in the case of the ferromagnets $\mathrm{EuO}$ and $\mathrm{EuS}$ [5]. For EuSe [6] as well as for the peculiar magnetic properties of EuTe and $\mathrm{Eu}_{x} S r_{1-x} \mathrm{Te}$ the introduction of an additional exchange term of the biquadratic type is required as will be detailed in this paper. The parameters $J_{1}$ and $J_{2}$ will of course change considerably if the Heisenberg sum given by (1) is complemented by such a term.

The hitherto unexplained magnetic features of EuTe can be summarized by the following points:

1. The low-temperature $\left(T \ll T_{N}\right)$ magnetization curve $M(B)$ of EuTe increases faster than linearly up to the critical field at $B_{c}=7.5 \mathrm{~T}$ where the second order phase transition into the paramagnetic state occurs $[7,8]$. The bilinear Heisenberg sum, however, predicts a linear behaviour, irrespective of how far the interaction reaches viz. how many terms are considered in (1).

2. The observed Néel line $T_{N}(x)$ of $\operatorname{Eu}_{x} \mathrm{Sr}_{1-x} \mathrm{Te}[9,10]$ decreases considerably faster than expected in a mean field model on dilution with strontium. Since $T_{N}(x)$ is proportional to $J_{2}$ no competing interactions are involved and therefore the simple linear relationship of the mean field theory

$T_{N}(x)=T_{N}(1) \cdot x$

should hold approximately, at least near $x=1$. Although an exact calculation taking into account the percolation threshold $x_{p}=0.13$ for nearest and next-nearest neighbor interactions will result in a somewhat modified slope, the observed slope of the normalized Néel line of 1.36 is appreciably larger than 1 .

3. The critical field $B_{c}(x, T=0)$ which is also a measure for the magnetic interaction strength, decreases more slowly than expected with dilution by Sr. In the composition range $0.8 \leqq x \leqq 1$ the observed slope of the normalized critical field line is 0.62 instead of 1 .

4. The critical exponent $\alpha$ of the specific heat of EuTe deviates strongly from the Heisenberg value $\alpha=-0.12$ with a crossover to a positive value of $\alpha=+0.38$ very close to $T_{N}$ [10]. On dilution with $\mathrm{Sr} \alpha$ decreases in a non-linear way, reaching the expected Heisenberg value at about $x=0.85$ and approaching finally a value of $\alpha \approx-1$ near to the critical concentration $x_{c}=0.48$ below which no long-range magnetic order exists [9].

5. Monte-Carlo simulations using the experimental values for $J_{1}$ and $J_{2}$ according to (2) arrive at too small values for $T_{N}$ and $B_{c}(T=0)$ for EuTe which again calls for some additional but up to now unspecified interaction [11].

It is the aim of the present work to show that the problems raised by the first four points above can be settled by the introduction of a biquadratic type of interaction. We are confident that also Monte-Carlo simulations will provide a more conclusive result by including a biquadratic interaction.

The separation of the total interaction into their bilinear and biquadratic contributions is achieved by an analysis of low-temperature magnetization curves from which the corresponding molecular fields viz. the external fields necessary to compensate them are obtained. It turns out that the relative weight of both interaction mechanisms changes with composition, a very favourable circumstance which allows to distinguish between the different effects both mechanisms have on the magnetic properties.

The paper is organized as follows. After a brief description of sample preparation and the experimental details of the magnetization and specific-heat measurements, the concentration dependence of the Néel temperature $T_{N}(x)$ and the critical field $B_{c}(x, T \rightarrow 0)$ will be discussed on the basis of the obtained values for biquadratic and bilinear interactions. We will show that the different slopes of both quantities near $x=1$ can be consistently explained using the molecular field constants evaluated from the curvature of the low-temperature magnetization curves. Then the particular type of magnetic phase diagram exhibiting a definite phase separation into antiferromagnetic and spin-flop phases as observed for $x<0.83$ will be presented. Finally it will be shown that also the concentration dependence of the critical exponent $\alpha$ of the specific heat may be related to the concentration dependence of the biquadratic exchange.

\section{Experimental}

$\mathrm{Eu}_{x} \mathrm{Sr}_{1-x} \mathrm{Te}$ single crystals were grown from stoichiometric compositions by sealing charges of 6 to $9 \mathrm{~g}$ in tungsten ampoules. The reaction of the elements to form $\mathrm{Eu}_{x} \mathrm{Sr}_{1-x} \mathrm{Te}$ compounds occurred during slow heating of the ampoules to about $700^{\circ} \mathrm{C}$ in either a tungsten-mesh heated vacuum furnace or by rf-heating. After this reaction the charges of all compositions were heated rapidly to above the melting point $\left(T_{m} \approx 2260^{\circ} \mathrm{C}\right)$ to about $2400{ }^{\circ} \mathrm{C}$ and were than cooled within about $3 \mathrm{~h}$ to $2100^{\circ} \mathrm{C}$ in a region with a slight temperature gradient. Subsequently the samples were kept in a region with a still stronger temperature gradient to allow for annealing and recrystallization processes.

This procedure yielded rubyred (EuTe) to slightly brownish (SrTe) transparent crystals of $13 \mathrm{~mm}$ diameter and up to $10 \mathrm{~mm}$ in length. In many cases the crystals exhibited subgrain boundaries and were brought to the desired sample size by cleaving along (100) planes. The stoichiometry as well as the composition $x$ were controlled mainly by susceptibility and saturation magnetization measurements which confirmed $g=2$ and $S=7 / 2$ within $\pm 1 \%$ for the $\mathrm{Eu}^{2+}$ ion.

Figure 1 shows that the lattice constants obey the Vegard rule quite well, a necessary criterion to verify a random solid solution of both constituents.

Magnetization measurements were performed with a Faraday magnetometer and specific heat measurements with an equipment using standard heat pulse techniques. Demagnetization effects were accounted for by correcting the internal field by $0.38 \mathrm{~T}$ for a spherical EuTe sample at saturation. The magnetocaloric effect (MCE) was measured with the sample coupled weakly to the heat bath. After a magnetic field increment $\Delta B$ of $50-150 \mathrm{mT}$, 


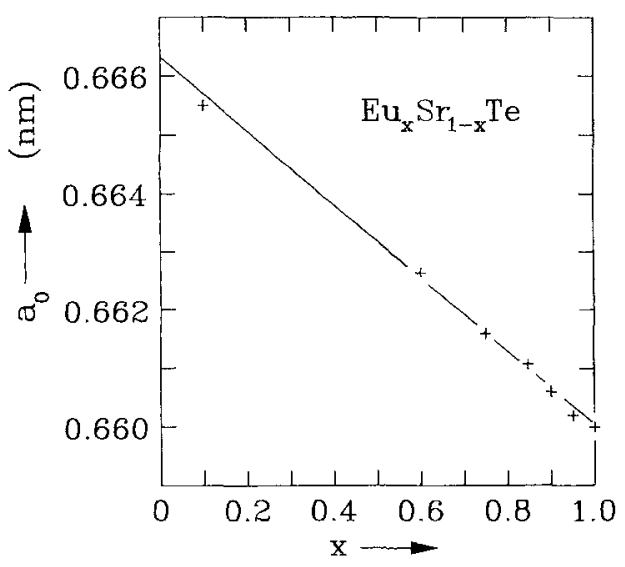

Fig. 1. Lattice parameter of $\mathrm{Eu}_{x} \mathrm{Sr}_{1-x} \mathrm{Te}$ samples as a function of $x$

the ensuing temperature change $\Delta T$ was measured. In this quasi-iso-thermal measurement, $T$ was allowed to return to the equilibrium value before the next $\Delta B$ step was applied. The field sweep rate was so low that eddycurrent heating was negligible.

The elastic neutron-scattering spectra were taken on powder samples using the $D 20$ diffractometer at the Institut Laue-Langevin Grenoble. The critical behaviour was studied on single crystals on the $D 10$ four circle diffractometer.

\section{Analysis of the low-temperature magnetization}

At low temperatures the sublattice magnetizations have developed their full values and are gradually turned from the antiparallel orientation for zero field into the parallel one for a sufficiently strong magnetic field. This gives us the opportunity to study the type of magnetic interaction by looking at the angular dependence of this process.

In the case of the usual Heisenberg interaction the hamiltonian is written as

$\mathscr{H}_{\text {ex }}=-2 J_{12} \mathbf{S}_{1} \cdot \mathbf{S}_{2}$.

In this expression we consider $S_{1}$ and $S_{2}$ as classical moments, which describe the sublattice magnetizations within a simplified two sublattice model. This seems to be justified considering the large spin quantum number of $\mathrm{Eu}^{2+}$. The interaction energy is than given by

$E_{\mathrm{ex}}^{1}=-2 J_{12} \cdot S^{2} \cdot \cos \varphi$

with $\varphi$ being the angle between both moments. For a biquadratic type of interaction we have accordingly

$E_{\mathrm{ex}}^{q}=-2 K_{12} S^{4} \cos ^{2} \varphi$.

In an applied magnetic field $B_{0}=\mu_{0} H_{0}$, which has to be converted to the internal field $B_{i}=\mu_{0} \cdot H_{i}$ according to $H_{i}=H_{0}-N \cdot M$ with $N$ the demagnetization factor, the angle $\varphi$ depends on the field strength and can be determined by a variational procedure. For simplicity we as- sume that both sublattice magnetizations are perpendicular to the field for $B_{i} \rightarrow 0$. Then the total potential energy amounts to

$$
\begin{aligned}
E= & -S_{1} g \mu_{B} \cdot H_{i} \cdot \cos \frac{\varphi}{2}-S_{2} g \mu_{B} \cdot H_{i} \cdot \cos \frac{\varphi}{2} \\
& -2 J_{12} S^{2} \cos \varphi-2 K_{12} S^{4} \cos ^{2} \varphi .
\end{aligned}
$$

Evaluating the energy minimum with respect to a variation of $\varphi$ and taking into account that the projection of both sublattice magnetizations along the field axis is measured, i.e., that the reduced magnetization $m=$ $M / M_{S}$ with $M_{S}$ as saturation magnetization is given by

$m=\cos \left(\frac{\varphi}{2}\right)$,

one obtains the relation between field $H_{i}$ and magnetization $m$ :

$H_{i}=-\frac{4 J_{12} S}{g \mu_{B}} m-\frac{8 K_{12} S^{3}}{g \mu_{B}} m\left(2 m^{2}-1\right)$.

Substituting the exchange parameters by their field analogues which are observed experimentally as the fields to be applied in order to compensate the molecular fields

$B_{\mathrm{ex}}^{1}=\frac{4 J_{12} \mu_{0} S}{g \mu_{B}}$ and $B_{\mathrm{ex}}^{q}=\frac{8 K_{12} \mu_{0} S^{3}}{g \mu_{B}}$

one gets for the internal field

$\mu_{0} \cdot H_{i}=B_{i}=-B_{\mathrm{ex}}^{1} \cdot m-B_{\mathrm{ex}}^{q} \cdot m\left(2 m^{2}-1\right)$.

Hence, if we plot the low temperature magnetization as $\mu_{0} \cdot H_{i} / m$ over $m^{2}$, a straight line should result, the slope of which is proportional to the biquadratic interaction strength. Such an analysis is shown in Fig. 2 for EuTe. Without biquadratic interaction $m \sim \mu_{0}{ }^{\circ} H_{i}$ would hold throughout and, therefore, a horizontal line should be observed in Fig. 2. As can be seen a fairly straight line

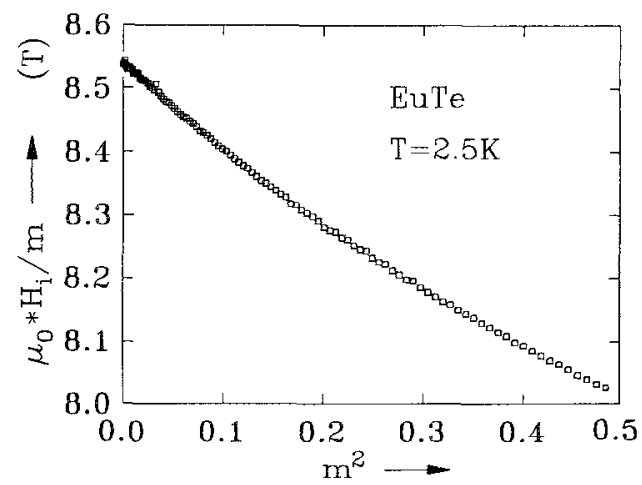

Fig. 2. Low-temperature magnetization curve of EuTe plotted as internal field devided by normalized magnetization vs. square of normalized magnetization. This plot gives the value of the molecular field as a function of $m^{2}$. The faster than linear increase of $m$ with $H_{i}$ due to a bilinear interaction term shows up in this representation by a decreasing interaction strength 
with negative slope results for EuTe (note the expanded vertical scale). The observed small deviations from linearity may have three reasons, domain rotation effects, zero point spin deviations and an unsaturated sublattice magnetization at the finite measuring temperature. We shall discuss these points separately.

- For samples with $0.83 \leqq x \leqq 1$ the low-temperature magnetization curves start first with a weak quadratic field dependence up to a field of $\sim 0.04 \mathrm{~T}$ [1]. This small field and magnetization range is not sufficiently resolved in Fig. 2. However, it might be necessary to shift the field scale relevant in Fig. 2 by $0.1 \mathrm{~T}$ due to this effect, which has however not bee done and this could give rise to an upturn by $\sim 1 \%$ in the small $\mathrm{m}^{2}$-range, as it is in fact observed in Fig. 2.

- The quantum-mechanical effect of a zero-point spinreduction in antiferromagnets leads to a gradual decrease of $m$ as the antiferromagnetic order is approached with decreasing magnetic field. Therefore, a qualitative behaviour like that displayed in Fig. 2 may be expected but the observed effect is much too large. Zero-point spin deviations have been estimated to be of the order of only 2 to $3 \%[8,12]$.

- The sublattice magnetization of EuTe is approximately $98 \%$ of its saturation value at $\mathrm{T}=2.5 \mathrm{~K}$ [8]. With increasing field it will tend towards $100 \%$ and this relative increase in $m$ will result in a small negative slope in Fig. 2 and adds thus to the two effects discussed above.

The non-linear field dependence of the magnetization curve which gives rise to the finite slope in Fig. 2 is however larger than estimated for the three mechanisms. Moreover this feature changes strongly with $x$ (see Fig. 3) and therefore cannot be explained by these three effects.

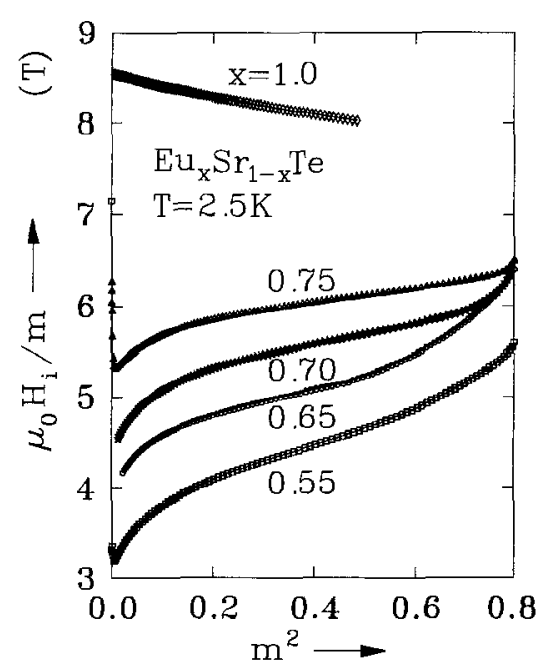

Fig. 3. Low-temperature magnetization curves of several $\mathrm{Eu}_{x} \mathrm{Sr}_{1-x} \mathrm{Te}$ samples plotted as internal field devided by normalized magnetization vs. square of normalized magnetization. At $x \approx 0.83$ the average slope of the curves changes from negative for $x>0.83$ to positive for $x<0.83$ due to a sign-change of the biquadratic interaction paramter. Deviations from linearity at small $m$ values (viz. $H_{i}$ values) are caused by the antiferromagnetic to spin-flop phase change. Curvatures above $m^{2} \approx 0.6$ occur because of the spin-flop to paramagnetic phase transformation
According to (11) the quantity $\mu_{0} \cdot H_{i} / m$ is limited to the values $-B_{\mathrm{ex}}^{1}+B_{\mathrm{ex}}^{q}$ for $m=0$ and $-B_{\mathrm{ex}}^{1}-B_{\mathrm{ex}}^{q}$ for $m=1$. This shows that both types of interaction add differently depending on whether the system is in its antiferromagnetic ground state $(m=0)$ or near to the critical field $B_{c}$ with a parallel orientation of both sublattice magnetizations $(m=1)$. In fact, Fig. 2 gives the actual molecular field as a function of $m^{2}$ and it can immediately be seen that both interactions add to a molecular field of $\sim 8.5 \mathrm{~T}$ in the antiferromagnetic ground state $(m=0)$ but they subtract in the ferromagnetic state $(m=1)$ to result in a critical field of $B_{c}(T \approx 0)=7.5 \mathrm{~T}$ as is observed experimentally (see Fig. 7). The decreasing antiferromagnetic interaction strength with increasing field is the reason why the magnetization increases faster than linearly (see Fig. 7). By convention, the fields occurring in (11) denote the internal molecular fields including their proper signs but in the following we prefer to give (as in Fig. 2) the experimental fields which must be applied to compensate the total molecular field which is always negative.

The situation changes considerably if the preceding analysis is performed for the diluted samples with $x<0.83$ as can be seen in Fig. 3. First, a positive slope of $\mu_{0} H_{i} / m$ vs. $\mathrm{m}^{2}$ is now observed over an appreciable $m$ range. Second, strong deviations from linearity are observed at low and high $\mathrm{m}^{2}$ values. These are due to magnetic phase transitions as will be detailed below. In contrast to the samples with $x>0.83$ a distinct phase transition between the antiferromagnetic (AF) and the spin-flop (SF) phase occurs at field values of about $0.3 \mathrm{~T}$. This phase transition manifests itself by a downward curvature of the curves in Fig. 3 at small fields viz. $m^{2}$-values. On the high field side close to $m^{2} \rightarrow 1$ an upward curvature indicates the phase transition at the critical field $B_{c}$ between the SF phase and the highly polarized paramagnetic $(\mathrm{P})$ phase. For samples with $x<0.83$ a straight-line

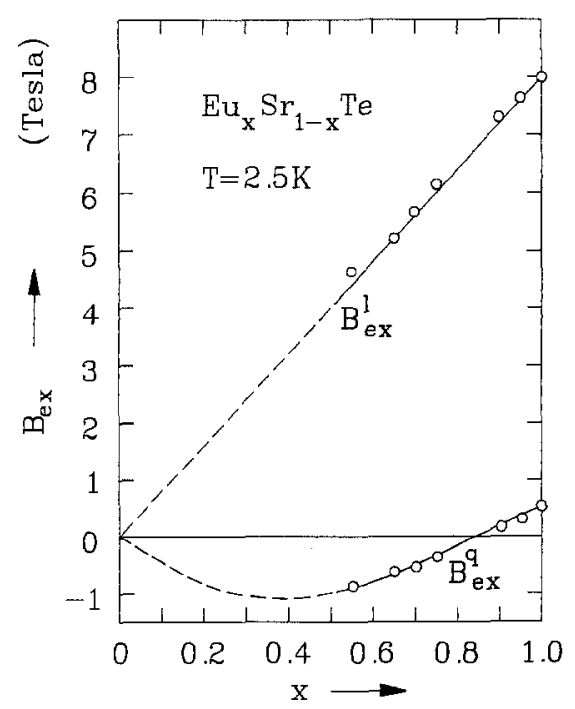

Fig. 4. Bilinear and biquadratic interaction fields $B_{e x}^{1}$ and $B_{\mathrm{ex}}^{q}$ as a function of composition $x$. The plot gives the external field values necessary to compensate the corresponding molecular ficlds in the antiferromagnetic ground state. The curve drawn through the $B_{\mathrm{ex}}^{q}$ data is an extrapolation including the origin as a necessary limit 

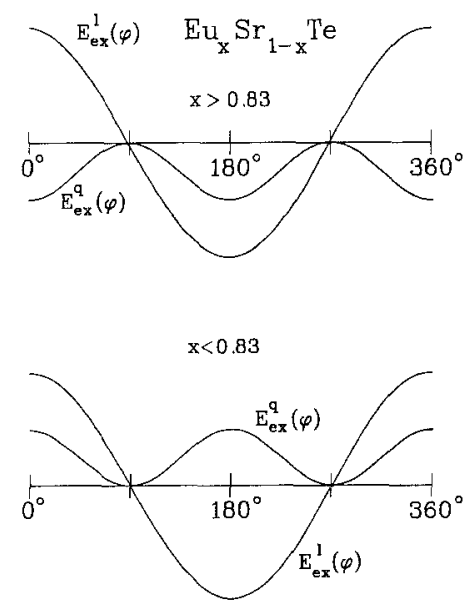

Fig. 5. Schematic visualization of the bilinear and biquadratic interaction potentials as a function of the angle between both sublattice magnetizations. $\varphi=180^{\circ}$ : antiferromagnetic orientation, $\varphi=0^{\circ}$ : ferromagnetic orientation of both sublattice magnetizations. For $x>0.83$ both types of interaction favour the antiferromagnetic orientation while for $x<0.83$ the energetic minimum of the biquadratic interaction is at the $90^{\circ}$ configuration

fit to the data according to (11) is only possible at intermediate magnetizations, i.e. sufficiently far away from both phase transitions. Figure 4 shows the bilinear and the biquadratic molecular field constants $B_{\mathrm{ex}}^{1}$ and $B_{\mathrm{ex}}^{q}$ resulting from the fit. While $B_{\mathrm{ex}}^{1}$ shows Vegard-type behaviour, $B_{\mathrm{ex}}^{q}$ exhibits a quite uncommon composition dependence with a sign change near $x \approx 0.83$. Thus, instead of decreasing linearly with dilution to zero at $x=0$, a strongly curved concentration dependence has to be assumed, i.e., $B_{\mathrm{ex}}^{q}(x)$ must pass through an absolute minimum before going to zero for $x=0$, as indicated by the dashed line in Fig. 4. Unfortunately, the analysis is restricted to $x \geqq 0.55$ since only those samples show a clear spontaneous long range magnetic order.

A non-linear behaviour of $B_{\mathrm{ex}}^{q}(x)$ will result if the coupling parameter $K_{12}$ is not a constant but depends on the composition $x$ [13]. Any linear $x$ dependence of $K_{12}$ gives an additional contribution proportional to $x(1-x)$ to the $B_{\mathrm{ex}}^{q}(x)$ dependence. In the present case we must infer a very strong concentration dependence of $K_{12}$ according to the strongly non-linear behaviour of $B_{\mathrm{ex}}^{q}(x)$.

The biquadratic interaction varies twice as fast with the angle $\varphi$ between the sublattice magnetizations as the bilinear interaction (cf. (5) and (6)). The corresponding molecular fields add therefore in a different way for the antiparallel and parallel orientation of both moments. This is illustrated schematically in Fig. 5, which visualizes the angular dependence of the interaction potentials. For $x>0.83$ both molecular fields superpose additively in the antiferromagnetic ground state $\left(\varphi=180^{\circ}\right)$ but they subtract in the parallel orientation at the critical field $\left(\varphi=0^{\circ}\right)$, and vice versa for the samples with $x<0.83$.

\section{Concentration dependence of the Néel temperature}

In order to test whether the exchange fields $B_{\mathrm{ex}}^{1}(x)$ and $B_{\mathrm{ex}}^{q}(x)$ obtained from the above analysis of low-tem-

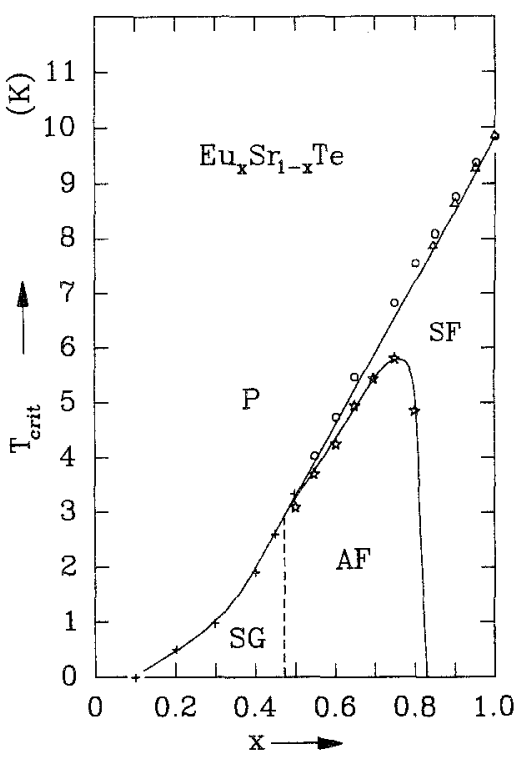

Fig. 6. Phase diagram of $\mathrm{Eu}_{x} \mathrm{Sr}_{1-x} \mathrm{Te}$. A genuine antiferromagnetic phase (AF) exists only for $0.48 \leqq x \leqq 0.83$. For $x>0.83$ the spinflop phase (SF) persists even for a vanishing magnetic field. Below $x_{c}=0.48$ a spin-glass state ( $\mathrm{SG}$ ) follows. Triangles: susceptibility results, open circles: maxima of specific heat, stars: AF-SF phase boundary extrapolated to zero field (compare Figs. 10, 12 and 13). Crosses are from [9]

perature magnetization curves describe the interactions in $\mathrm{Eu}_{x} \mathrm{Sr}_{1-x} \mathrm{Te}$ appropriately, we may employ them to explain the composition dependence of $T_{N}(x)$ and $B_{c}(x)$. Both quantities are a measure for the interaction strength but the former in the case of an antiferromagnetic spin arrangement and the latter in the case of a ferromagnetic spin arrangement. In both situations different interaction strengths apply. In particular, in the antiferromagnetic ground state we have to add $B_{\mathrm{ex}}^{1}(x)$ and $B_{\mathrm{ex}}^{q}(x)$ given in Fig. 4 to result in a quantity proportional to the Néel line $T_{N}(x)$, at least in the molecular field approximation. Approximating the linear part of the experimental $B_{\mathrm{ex}}^{q}(x)$ data on the high-concentration side by $B_{\mathrm{ex}}^{q}(x)=$ $3.33 x-2.83$ and using $B_{\mathrm{ex}}^{1}(x)=8.0 x$ (with $\mathrm{T}$ as field unit) we obtain for the normalized sum of both exchange fields

$B_{\mathrm{ex}}^{a f}(x) / B_{\mathrm{ex}}^{a f}(1)=1.33 x-0.33$.

Earlier results $[9,10]$ for the Néel line $T_{N}(x)$ complemented by new data obtained in this work are shown in Fig. 6. Here the normalized Néel line can be described in its linear part on the high-concentration side by

$T_{N}(x) / T_{N}(1)=1.36 x-0.36$.

The agreement between (12) and (13) is surprisingly good. Within this analysis we understand the deviation of the slope of the normalized Néel line from unity mainly as a consequence of the biquadratic interaction $B_{\mathrm{ex}}^{q}(x)$ which has, normalized to its $B_{\mathrm{ex}}^{q}(1)$ value, a slope of 6.7 such that any admixture of small contributions of biquadratic interaction changes the slope for the total magnetic interaction considerably. To a lesser extent, effects due to 
percolation and competing interactions may also increase the slope of the $T_{N}(x) / T_{N}$ (1) line with respect to unity borne out by the mean-field theory but these effects are difficult to calculate quantitatively [14] and seem to be of minor importance here.

\section{Concentration dependence of the critical field}

A further consistency test for the proposed separation of the total interaction into bilinear and biquadratic contributions is provided by the composition dependence of the critical field $B_{c}(x)$ in the limit $T \rightarrow 0$. The critical field is proportional to the antiferromagnetic interaction strength in the state of a forced ferromagnetic orientation of both sublattice magnetizations. In this configuration both interaction types counteract and therefore the critical field should be proportional to

$B_{c}(x) \approx B_{\mathrm{ex}}^{1}(x)-B_{\mathrm{ex}}^{q}(x)$

or, with $B_{\mathrm{ex}}^{1}(x)=8 x$ and $B_{\mathrm{ex}}^{q}=3.33 x-2.83$ (again in $\mathrm{T}$ ):

$B_{c}(x) / B_{c}(1)=0.62 x+0.38$.

In other words, the $x$ dependence of $B_{c}(x) / B_{c}(1)$ is reduced by the same amount as the $x$ dependence of $T_{N}(x) / T_{N}(1)$ was increased.

In order to evaluate the critical fields we employ the differentiated magnetization curves as shown in Fig. 7, since for $x<0.83$ the anomalies associated with the phase transition at $B_{c}(x)$ are so weak that they can hardiy be detected on the magnetization curve itself. While for the samples with $x>0.83$ the magnetization curves show a visible change in slope at $B_{c}$ and, therefore, nearly a discontinuity in the $\mathrm{d} m / \mathrm{d} B$ curve, for the samples with $x<0.83$ the first derivative of the magnetization curve shows only a change in slope at $B_{c}$ but the magnetization curve itself lacks any marked anomaly at $B_{c}$. The magnetocaloric effect is here a superior method since it dis-

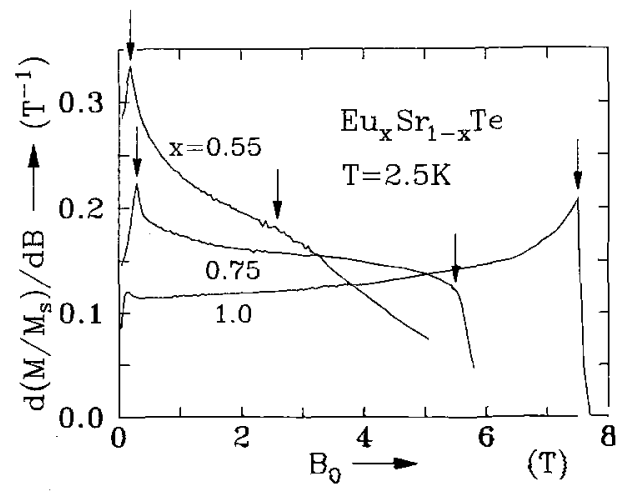

Fig. 7. First derivatives of low-temperature magnetization curves. For $x<0.83$ a sharp peak at $B_{0} \approx 0.3 \mathrm{~T}$ indicates the antiferromagnetic to spin-flop phase transition of second order. The phase transition into the paramagnetic state shows up as a discontinuity for EuTe but as a progressively weaker change in slope for samples with $x<0.83$. Critical field values are marked by arrows

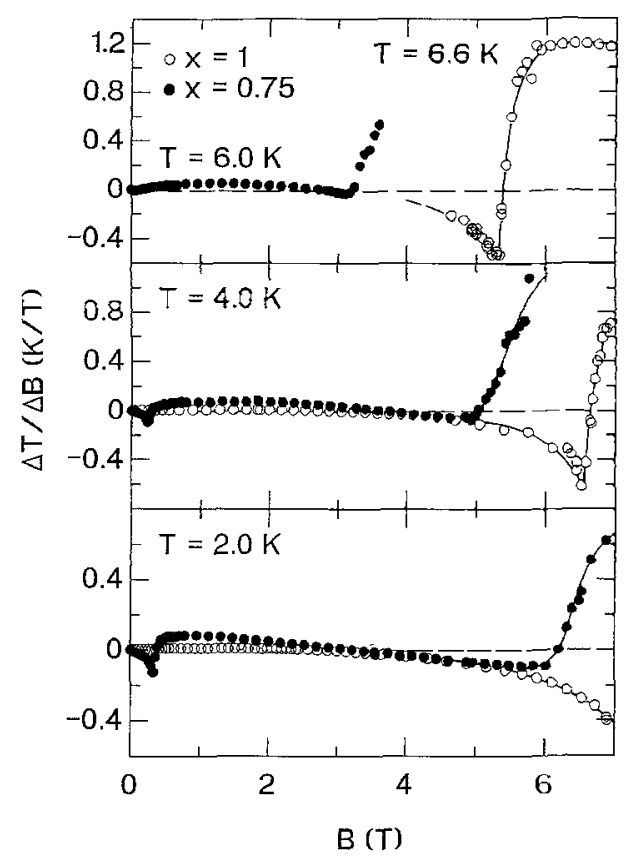

Fig. 8. Magnetocaloric effect $\Delta T / \Delta B$ vs. magnetic field $B$ for $0 \leqq B \leqq 7 \mathrm{~T}$ for concentrations $x=0.75$ (closed circles) and $x=1$ (open circles) at three different temperatures. For both concentrations the transition to the saturated paramagnetic state is visible by a sharp minimum at fields $B>3 \mathrm{~T}$

plays rather sharp features at $B_{c}$ for all temperatures as is shown in Fig. 8 for $x=0.75$ and $x=1$. These results will be discussed in more detail below.

A second difference between the two concentration ranges above and below $x=0.83$ concerns a sharp peak seen at $B \leqq 0.3 \mathrm{~T}$ in the $\mathrm{d} m / \mathrm{d} B$ curves for the samples with $x<0.83$ (Fig. 7). As will be outlined below too, this phenomenon marks a critical phase transition between the antiferromagnetic phase and the spin-flop phase which is not seen for the sample with $x>0.83$. On the other hand, the small anomaly seen in the EuTe curve $(x=1)$ at $B=0.05 \mathrm{~T}$ is attributed to domain rotations which is confirmed by the absence of any structure in measurements of the magnetocaloric effects (see below).

Figure 9 shows the observed critical fields $B_{c}(x)$ at $T=2.5 \mathrm{~K}$ as obtained from magnetization measurements (open squares). In the small composition interval $0.85 \leqq x \leqq 1$ the slope of the $B_{c}(x)$-curve does in fact correspond to the value given by (15) as is indicated by the straight line but for smaller concentrations the situation changes considerably because of the occurrence of an intervening AF phase. The reduced slope of 0.62 for the normalized critical field $B_{c}(x) / B_{c}(1)$ is even better confirmed by the specific heat results either extrapolated to $T=0$ (circles) or at $T=2.5 \mathrm{~K}$ (stars). However, magnetic and caloric results deviate systematically in particular at low temperatures and small compositions for reasons which are not well understood. One difficulty may be due to the fact that in the magnetization measurements the associated anomalies are small features on a large background signal which introduces ambiguities in the choice of the correct base-line. 


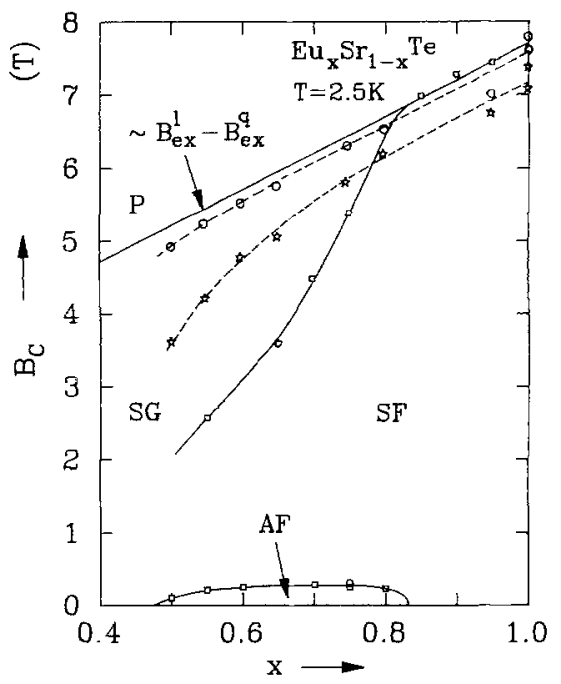

Fig. 9. Critical field values observed at $T=2.5 \mathrm{~K}$ with magnetization measurements (open squares) and with specific heat measurements (stars). Open circles give the critical field from specific heat extrapolated to zero temperature. Although both methods give quantitatively different results, in particular in the low $T$, low $x$ and high $B$ range, the initial slope $B_{\mathrm{ex}}^{1}-B_{\mathrm{ex}}^{q}$ is well reproduced in all cases. For the AF-SF phase boundary the magnetocaloric effect (open circle at $x=0.75$ ) and the magnetization measurements are in better agreement

\section{Antiferromagnetic to spin-flop phase boundary}

For the samples with $x>0.83$ bilinear and biquadratic interaction both favour the antiferromagnetic order (see Fig. 5). This accord between both interactions seems to be the reason that in EuTe the AF-SF phase line is depressed to $B_{0}=0$ and that the system stays always in the SF phase once a magnetic field has been applied. This view-point is at variance with earlier suggestions of a spin-flop transition occuring in EuTe [1, 15], but it conforms to computer simulations [11] which proved that the dipole-dipole anisotropy is not strong enough to result in an AF-SF transition at finite fields. In fact, the differentiated magnetization curves (Fig. 7) show some anomaly but this is not associated with a critical phase transition as could be confirmed in the present work by the absence of a magnetocaloric effect in small magnetic fields. Apparently, the dipole-dipole anisotropy is not strong enough to result in an AF-SF phase transition at finite fields [11], but gives rise only to non-critical magnetization or domain rotations. For $x<0.83$, however, the biquadratic interaction has changed its sign tending to a perpendicular sublattice orientation, while the dominating bilinear interaction is still in favour of the antiferromagnetic order. Such a conflicting situation introduces an anisotropy and this shifts the AF-SF phase line to finite fields. The associated spin-flop field $B_{\mathrm{AF}-\mathrm{SF}}$ is in fact of the order of the biquadratic molecular field $B_{\mathrm{ex}}^{q}$ and reaches values of $0.3 \mathrm{~T}$ for $T \rightarrow 0$.

At the boundary of this composition-induced genuine AF phase at finite fields the magnetic properties change considerably as a function of the magnetic field such that the SF phase has already much similarity with the satu-
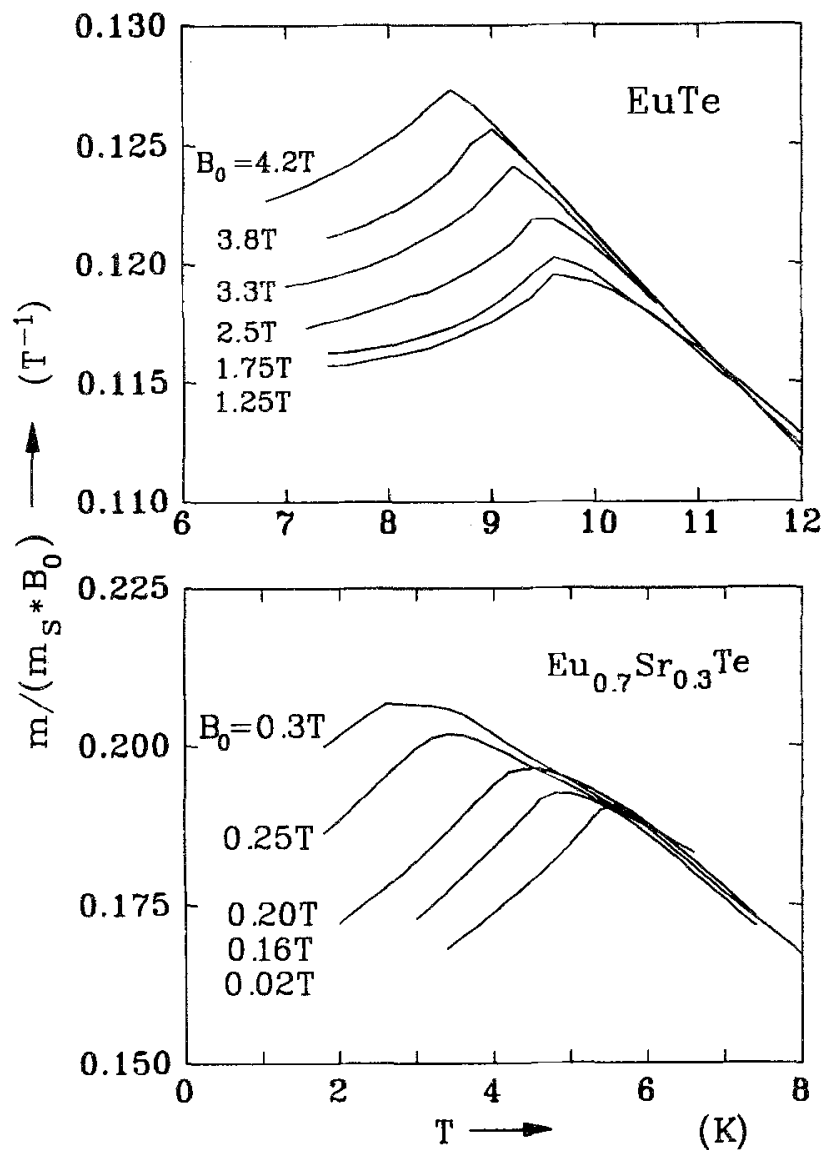

Fig. 10. Temperature scans of magnetization at different field values for EuTe (upper part) and $\mathrm{Eu}_{0.7} \mathrm{Sr}_{0.3} \mathrm{Te}$ (lower part). In spite of a qualitative similarity of both data sets the maxima of the EuTe curves give the phase boundary between spin-flop and paramagnetic phase while for the sample with $x=0.7$ these maxima are pertinent to the antiferromagnetic to spin-flop transition. Note the much stronger temperature shift of the maxima with field for the $x=0.7$ sample

rated paramagnetic phase for $x<0.83$ in contrast to $x>0.83$ and, as a consequence, no strong anomalies do occur anymore at $B_{c}(x)$ for $x<0.83$. This makes the $B_{c}(x)$ line hard to observe and leads to considerable ambiguities in the evaluation of this phase boundary (cf. Fig. 7).

We should, however, bear in mind that both critical fields $B_{c}(x, T)$ and $B_{\mathrm{AF}-\mathrm{SF}}(x, T)$ are functions of $x$ and $T$. As a consequence of the similarity between SF phase and saturated paramagnetic phase for $x<0.83$ the magnetization phenomena along $B_{c}(x, T)$ for $x>0.83$ resemble those along $B_{\mathrm{AF}-\mathrm{SF}}(x, T)$ for $x<0.83$ if we cross both phase boundaries at constant $x$ as a function of temperature. This is illustrated in Fig. 10 which compares susceptibility curves normalized to the saturation magnetization and the applied field for EuTe and $\mathrm{Eu}_{0.7} \mathrm{Sr}_{0.3} \mathrm{Te}$, respectively. Both sets of curves seem to be reminiscent of transitions into the antiferromagnetic state but in spite of a qualitative similarity they pertain to different phase transitions. In the case of EuTe the maxima of the curves mark the phase boundary between the paramagnetic and the SF phase. From the normalization of the curves it can immediately be seen that the magnetization increases 
faster than linearly with field. In the case of the $\mathrm{Eu}_{0.7} \mathrm{Sr}_{0.3} \mathrm{Te}$ sample the maxima of the curves have to be identified with the phase transition between the SF and the AF phase. The fact that another phase boundary is sampled becomes evident from the observation that the maxima of the $\mathrm{Eu}_{0.7} \mathrm{Sr}_{0.3} \mathrm{Te}$ curves shift much faster to smaller temperatures with increasing field, extrapolating to a field value of only $B_{\mathrm{AF}-\mathrm{SF}}(x=0.7, T \rightarrow 0)=0.35 \mathrm{~T}$ instead of $B_{c}(x=1, T \rightarrow 0)=7.5 \mathrm{~T}$. A change by a factor of $\approx 20$ in the critical field induced by a dilution of only $30 \%$ would not be reasonable without assuming that other fundamental changes have taken place in the system and as such we identify the rise of a phase change between $\mathrm{AF}$ and SF phase for $x \leqq 0.83$. The curves for $\mathrm{Eu}_{0.7} \mathrm{Sr}_{0.3} \mathrm{Te}$ in Fig. 10 follow the same sequence as those for EuTe with the curve measured at the highest field having the largest magnetization. This is because with the small fields applied we are too close to the AF-SF phase transition and therefore the relative decrease of magnetization as it becomes apparent at high fields is not yet perceptible.

The differentiated low-temperature magnetization curves for the samples with $x<0.83$ like in Fig. 7 show the two phase transitions AF-SF and SF-P in a particularly clear fashion. At the AF-SF transition a peak results in the differentiated magnetization curve which means that $m$ vs. $B_{0}$ exhibits a smeared step, a behaviour which is tpyical for an antiferromagnetic to spin-flop transition of second order. At the spin-flop to paramagnetic transition the slightly negative slope of the $\mathrm{d} m / \mathrm{d} B$ vs. $B_{0}$ curve changes to a much stronger decrease. It is clear that due to this comparatively weak anomaly the SF-P transition cannot be located with the same precision as the AF-SF transition. Here the specific heat provides a somewhat clearer picture [10] but with systematic deviations with respect to the magnetization results.

To support the conclusions drawn from the magnetization measurements, the magnetocaloric effect (MCE) of a few samples with $x \geqq 0.75$ has been examined. Figure 8, as mentioned above, displays representative results for two samples with $x=0.75$ and $x=1$ at three temperatures below the Néel temperature $T_{N}(x)$ up to the highest experimentally attainable magnetic field of $7 \mathrm{~T}$. In the magnetocaloric effect where $\Delta T / \Delta B$ is measured as function of the applied magnetic field $B$ a second order phase transition is located at the inflection point from a positive to a negative curvature. The thermal measurements give somewhat higher values for the critical fields than the magnetization measurements do, as discussed above.

Figure 11 shows the low-field MCE data up to $0.6 \mathrm{~T}$ with higher resolution. The data of the sample with $x=0.75$ show pronounced features which are identified with the AF-SF transition observed in the magnetization measurements in Fig. 7 and 10. For $T=6.0 \mathrm{~K}$ the anomaly is smeared out so that the inflection point could not be located with high precision. Therefore, we chose the field value of the minimum as transition field $B_{\mathrm{AF}-\mathrm{SF}}$. In contrast to this obvious AF-SF phase transition for $x=0.75$, the curves of $x=1$ are featureless within our resolution.

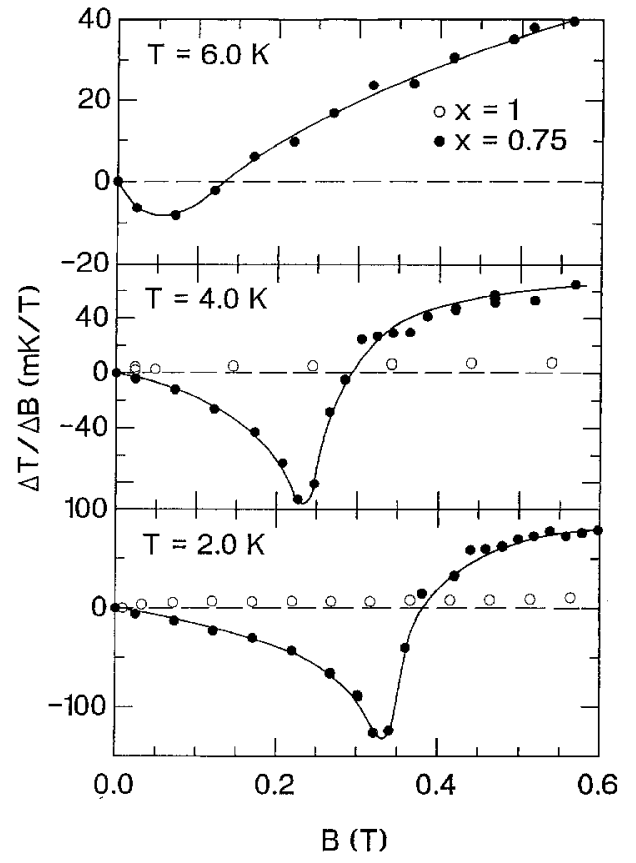

Fig. 11. Magnetocaloric effect $\Delta T / \Delta B$ vs. magnetic field $B$ for $0 \leqq B \leqq 0.6$ T for concentrations $x=0.75$ and $x=1$ at three different temperatures. For $x=0.75$ the AF-SF transition is observable for all temperatures. In the curve for $x=1$ no anomaly can be seen. For temperatures $T>2 \mathrm{~K}$ the magnetocaloric effect of the sample with $x=1$ was zero up to $B \approx 1 \mathrm{~T}$ within our resolution

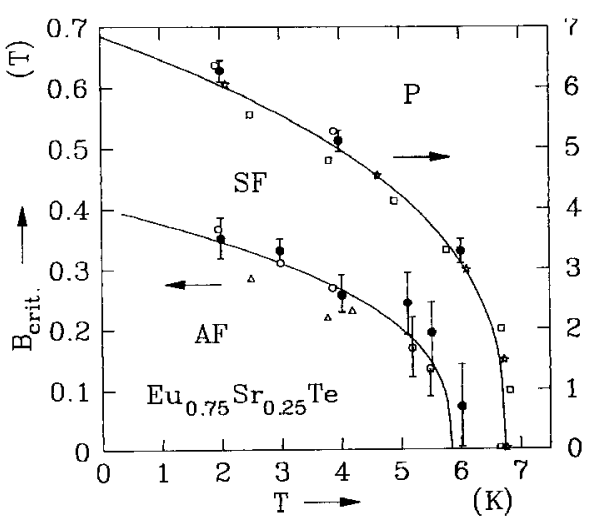

Fig. 12. Magnetic phase diagram of $\mathrm{Eu}_{0.75} \mathrm{Sr}_{0.25} \mathrm{Te}$ showing antiferromagnetic (AF), spin-flop (SF) and paramagnetic phase (P). Note the different field scales for AF-SF and SF-P boundaries. Triangles and squares are magnetization data either at constant field or at constant temperature. Stars are data from specific heat measurements while open and closed circles indicate magnetocaloric anomalies as obtained from measurements on two different samples

The twofold anomalies of Figs. 7 and 8 and critical field values obtained from measurements like in Fig. 10 are combined to construct the magnetic phase diagram in Fig. 12 giving the phase boundaries of $\mathrm{AF}, \mathrm{SF}$ and $\mathrm{P}$ phase. Because of the very different field values for the $B_{c}(T)$ and $B_{\mathrm{AF}-\mathrm{SF}}(T)$ phase lines, both are given in scales which differ by a factor of 10 .

MCE measurements were repeated on a second sample (open and filled circles in Fig. 12, respectively) yielding 
the same transition fields within the error bars. In addition, samples with $x=0.85$ and $x=0.90$ have been investigated, and virtually the same behaviour as for $x=1$ was observed. These results exclude the existence of an AF-SF transition within the investigated field range for $x \geqq 0.85$.

The most interesting aspect of the phase diagram in Fig. 12 is that the three magnetic phases do not coexist and that the spin-flop phase apparently is the zero-field ground state in a small temperature interval. This peculiar behaviour can be understood qualitatively by the fact that for $x<0.83$ the biquadratic interaction prefers, according to Figs. 4 and 5, a perpendicular orientation of both sublattice magnetizations, but the bilinear one an antiferromagnetic order and, therefore, the tilted configuration of the spin-flop state represents a compromise. However, at lower temperatures, the antiferromagnetic order prevails, but only for a limited $T$ and $B_{0}$ interval. There are stringent theoretical arguments supporting the geometrical construction of the phase diagram depicted in Fig. 12. As we have shown, both phase lines $B_{c}(x, T)$ and $B_{\mathrm{AF}-\mathrm{SF}}(x, T)$ are of second order. If all three phases $\mathrm{AF}$, $\mathrm{SF}$ and $\mathrm{P}$ would coexist at some point, three second order phase lines would meet which is thermodynamically forbidden as has been pointed out by Yip et al. [16]. The only bicritical point possible is one where two phase lines of second order and one phase line of first order meet.

At this point we should come back to the magnetic phase diagram given in Fig. 6, which compiles the phase transitions observed for $B_{0} \rightarrow 0$ as they were taken from the maxima in the susceptibility measurements (triangles), from specific heat measurements (circles) and from the $B_{\mathrm{AF}-\mathrm{SF}}(T)$ curves extrapolated to zero field (see lower section of Fig. 10). It is appropriate to identify the Néel line $T_{N}(x)$ as the phase transition between the paramagnetic and the spin-flop phase throughout because there is no AF-SF phase transition at finite fields for $x>0.83$ which means that the system remains always in the SF phase even if returned to field zero after a magnetization process. Some weak anomalies seen at about $0.04 \mathrm{~T}$ in the differentiated magnetization curves for the samples with $x>0.83$ indicate magnetization rotations within the (111) planes [1, 15] but these are continuous i.e. non-critical processes as is revealed by the absence of MCE anomalies. Only for $x<0.83$ a genuine AF-phase emerges due to a sufficiently strong anisotropy provided by the perpendicular coupling of the biquadratic interaction but this AF phase does not approach the $T_{N}(x)$ phase line for topological reasons since a vortex of three phase lines of second order is forbidden. At the critical composition $x_{c}=0.48$ where the long-range magnetic order breaks down with a finite ordering temperature AFSF and SF-P phase lines seem to converge but this point must be considered as a singular one. We should note that both phase diagrams given in Figs. 6 and 9 are topologically equivalent. In both cases the $B_{c}(x)$ and $T_{N}(x)$ separate the $\mathrm{SF}$ and $\mathrm{P}$ phase and for $x<0.83$ an AFphase emerges which is restricted to small field values in the $B_{c}(x, T)$ phase diagram, but approaches $T_{N}$ closely in the $T_{N}(x)$ phase diagram.

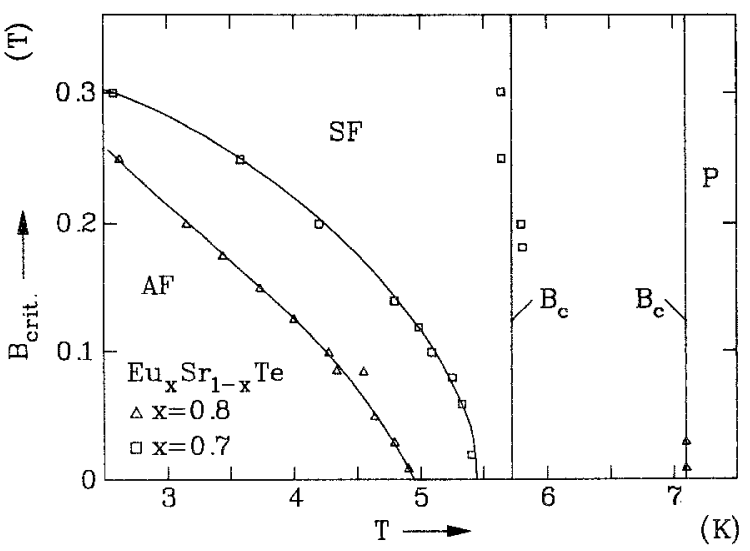

Fig. 13. Magnetic phase diagrams for $\mathrm{Eu}_{0.8} \mathrm{Sr}_{0.2}$ Te (triangles) and $\mathrm{Eu}_{0,7} \mathrm{Sr}_{0,3} \mathrm{Te}$ (squares) determined from magnetization measurements. The paramagnetic (P), to spin-flop (SF) transition appears here as a vertical line. The SF-AF phase boundary is much more field dependent and has been determined from the maxima of temperature scans at different field values like those shown for $x=0.7$ in Fig. 10

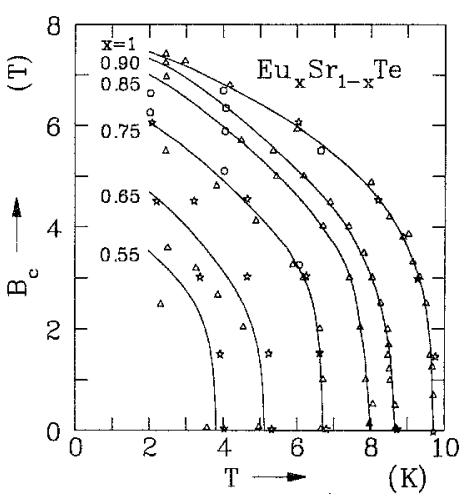

Fig. 14. Temperature dependence of the critical field $B_{c}$ (phase boundary between SF and $\mathrm{P}$ phase), for some $\mathrm{Eu}_{x} \mathrm{Sr}_{1-x} \mathrm{Te}$ samples. Triangles are data from magnetization measurements, circles from magnetocaloric effect measurements and stars from specific heat measurements. For smaller Eu-concentrations progressively stronger systematic deviations between the caloric and magnetic measurement occur. Solid lines are guides to eye only

In Fig. 13 we give two further examples of magnetic phase diagrams for $x=0.7$ and $x=0.8$, but this time with the same scale for both phase boundaries. The sample with $\mathbf{x}=0.8$ shows the widest temperture interval of the spin-flop phase at $B_{0}=0$ which extends over more than $2 \mathrm{~K}$. In the case of $x=0.7$ (open squares) both critical lines $B_{c}(x, T)$ and $B_{\mathrm{AF}-\mathrm{SF}}(x, T)$ approach each other very closely for $B_{0} \rightarrow 0$. Comparison of the extent of the AFphase as function of $x$ shows that this phase is suppressed more strongly for $x=0.8$ suggesting that it will shrink into the origin for $x \rightarrow 0.83$ as indicated in Fig. 6 .

Figure 14 displays critical field curves $B_{c}(x=$ const., $T$ ). It is obvious that the three curves with $x=1.0,0.9$ and 0.85 do not scale, since the critical field values for $T \rightarrow 0$ decrease much less with dilution than do the Néel temperatures at $B_{0} \rightarrow 0$. For the curves with $x=0.55$ and 
0.65 the low-temperature values of the caloric and magnetic measurements differ systematically probably, because this transition appears only as a weak feature in the magnetization curves.

\section{Neutron diffraction experiments}

Up to now we have tacitly assumed that the antiferromagnetic state of EuTe is preserved on dilution with $\mathrm{SrTe}$ down to $x_{c}=0.48$. To verify this we have performed neutron diffraction measurements on powder samples. Figure 15 shows two difference spectra which give the magnetic diffraction intensities. Both spectra are qualitatively the same and agree well with the one of [2]. They show only all-odd indexed reflections on the basis of a magnetic unit cell which is doubled along all three space directions with respect to the chemical unit cell. This selection rule is typical for the $\mathrm{MnO}$ magnetic structure [2].

While the selection rule clearly indicates that the typ II antiferromagnetic structure is present for both, the pure and the diluted sample, a unique determination of the moment direction is not possible from powder data alone. On the other hand, systematic single crystal neutron diffraction data on EuTe are difficult to obtain due to the large absorption cross section of Eu. The problem with powder data has been discussed by a number of authors after the first magnetic structure determination for $\mathrm{MnO}$ [36-39]. In fact, for the typ II antiferromagnetic order, the sublattice magnetization direction can be different for all four sublattices represented by the spins in $(0,0,0),(1 / 2,1 / 2,0),(0,1 / 2,1 / 2)$ and $(1 / 2,0,1 / 2)$. Due to the powder averaging, the changes in the diffraction patterns with moment directions are only minor. Even if a collinear type IIA structure [37] is assumed, the magnetic unit cell will have rhombohedral symmetry and only the angle between the moment direction and the rhombohedral axis (i.e. the cubic [111] direction) can be determined [17]. To obtain the best possible information, we have calculated magnetic intensities for various collinear and canted models and compared the calculated to the observed intensities. For both samples we get a reasonable fit assuming a collinear structure with the moments lying in the (111) planes. As pointed out in [38] this structure is favoured since it minimizes the dipolar energy. However, Henley [40] has discussed the ground state selection in fcc antiferromagnets on the basis of fluctuating interactions. He proposes a collinear structure for the pure samples and a canted structure for the diluted case. From our data alone, we can not decide upon this prediction: for the $x=0.65$ sample a structure might be present where the moments lie within the (111) planes, but are not collinear.

On two single crystal samples with $x=1$ and $x=0.65$ we have measured the temperature dependence of the 311 magnetic reflection in order to evaluate the critical exponent $\beta$ of the sublattice magnetization. In the case of the sample with $x=0.65$ a particularly careful subtraction of the critical diffuse scattering was necessary to separate the magnetic Bragg intensity from background. Figure 16 gives a log-log plot of the magnetic scattering intensities normalized to the extrapolated saturation value for $T \rightarrow 0$

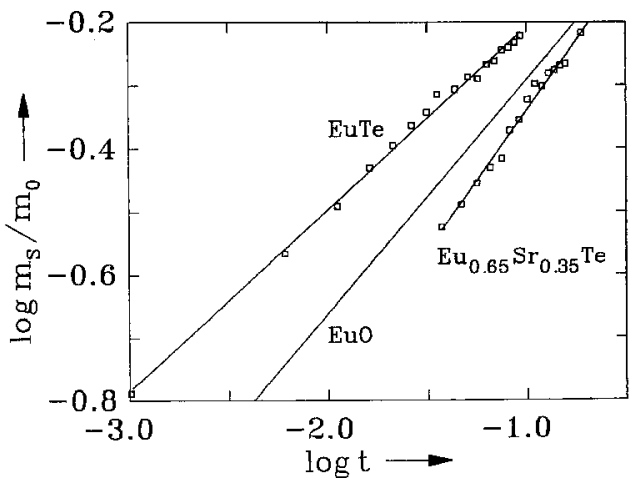

Fig. 16. Log-log plot of sublattice magnetization normalized to the extrapolated value for $T \rightarrow 0$ vs. reduced temperature $t=\frac{T_{N}-T}{T_{N}}$. Squares are data from neutron diffraction experiments. The critical behaviour of the spontaneous magnetization of EuO taken from [18] has been added as a reference of a system showing $3 d$-Heisenberg critical behaviour
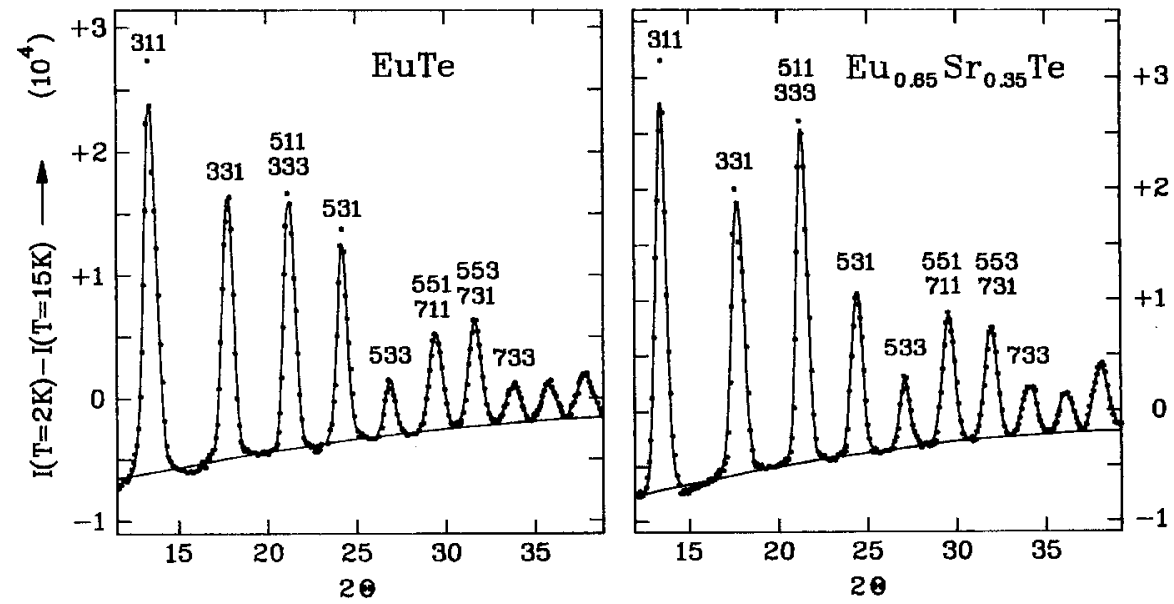

Fig. 15. Magnetic neutron diffraction data obtained from the intensity differences between spectra taken at $T=2 \mathrm{~K}$ and $T=15 \mathrm{~K}$. The magnetic structure of the diluted sample appears to be unchanged with respect to EuTe. The relative intensities of the magnetic reflections are in both cases consistent with a moment orientation confined to the 111 plane in which equal orientations are found 
vs. the reduced temperature $t=\frac{T_{N}-T}{T_{N}}$. For comparison the critical behaviour of the spontaneous magnetization of EuO taken from [18] has been included.

The critical exponent $\beta=0.29 \pm 0.01$ obtained for EuTe is considerably smaller compared to the theoretical prediction for the $3 d$-Heisenberg model $\sim 0.36$ [19]. For the ferromagnet EuO, on the other hand, $\beta=$ $0.368 \pm 0.005$ has been evaluated [18] which is in perfect agreement with the $3 d$-Heisenberg prediction. Due to the larger problems with the critical diffuse scattering, the $\beta$ value for the $x=0.65$ sample is less reliable but the obtained value of $\beta=0.44 \pm 0.03$ is now larger than expected. We shall discuss these results together with the critical exponent of the magnetic specific heat which was investigated in more detail.

\section{Critical behaviour of the magnetic specific heat}

In completion of recent studies of the critical behaviour of the magnetic specific heat $c(T)$ on $\mathrm{Eu}_{x} \mathrm{Sr}_{1-{ }_{x}} \mathrm{Te}$ samples [10] we measured $c(T)$ of two further samples with $x=0.85$ and $x=0.90$. Experimental details of the $c(T)$ measurements as well as the data analysis are given in [10].

The pure system EuTe shows a slightly positive exponent $(\alpha=+0.0085)$ in a limited temperature interval not too close to $T_{N}(0.01</ t /<0.06)$. On approaching $T_{N}$ more closely a crossover to a large positive value of $\alpha=+0.38 \pm 0.1$ is observed. This state of affairs is confirmed with dilatometric measurements [20]. The critical behaviour of EuTe deviates thus strongly from the $3 d$ Heisenberg model, which predicts $\alpha=-0.12$ [19]. Interestingly, the crossover to large positive $\alpha$-values has disappeared already for $x=0.95$.

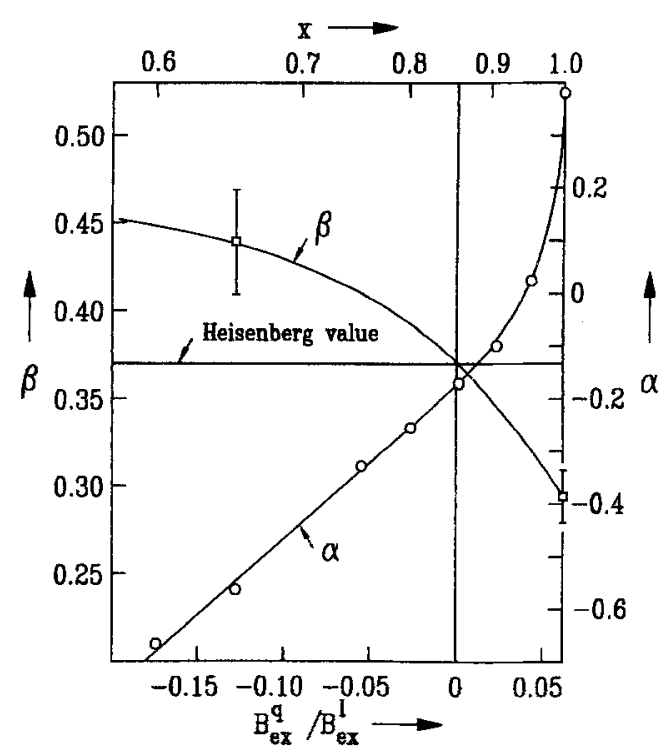

Fig. 17. Critical exponent $\alpha$ of the magnetic specific heat (righthand scale) as a function of the ratio $B_{\mathrm{ex}}^{q} / B_{\mathrm{ex}}^{1}$ (lower scale) viz. composition (upper scale) $\alpha$ is close to the Heisenberg value for $B_{\mathrm{ex}}^{q}=0$. The same seems to hold for $\beta$, the critical exponent of the sublattice magnetization (open squares, left-hand scale)
It is tempting to relate the anomalous values of the critical exponent $\alpha$ to the strength of the biquadratic interaction. In Fig. 17 we have therefore plotted the experimental $\alpha$ values vs. the ratio of the biquadratic and bilinear molecular field constants $B_{\mathrm{ex}}^{q} / B_{\mathrm{ex}}^{1}$. The upper scale gives the corresponding Eu concentration $x$. Also included are the two data points for the critical exponent $\beta$ of the sublattice magnetization obtained with the neutron diffraction measurements (left-hand scale).

The main reason to believe that the deviations of $\alpha$ from the Heisenberg value $\alpha=-0.12$ may be due to the presence of the biquadratic interaction is provided by the observation that $\alpha$ assumes a value of about -0.12 near to that concentration where $B_{\mathrm{ex}}^{q}$ vanishes $(x \approx 0.83)$. This does, however, not hold correctly, but considering that we are dealing with a diluted antiferromagnet we must be aware of the possibility that $\alpha$ may decrease additionally by the phenomenon of site disorder as it was found for $\mathrm{Eu}_{x} \mathrm{Sr}_{1-x} \mathrm{~S}$ [21], a diluted ferromagnetic system in which a "normal" $\alpha$-value is observed for the undiluted case $x=1$ [22]. The dilution dependence of $\alpha$ observed there is in qualitative accord with the Harris criterion [35], which tells that the critical exponents do change with dilution if $\alpha$ is finite for the pure system.

The two data points for $\beta$ seem to be compatible with this picture i.e. that the Heisenberg predictions are nearly fulfilled if $B_{\mathrm{ex}}^{q}=0$. Note that the solid lines drawn through the experimental points are guides to the eye only. A definite conclusion concerning $\beta$ has, of course, to wait for further measurements.

Generally, the critical behaviour at a magnetic transition depends only on a few parameters of the system such as the dimensionality, the symmetry of the order parameter, e.g., the number of its components, and the range of the interaction. It is thus not clear if and how the biquadratic interaction delineated in the present work affects the universality class.

The most likely view appears to be that EuTe is close to a phase transition of first order which complies with an enhanced $\alpha$ value and a too small $\beta$ value. A first order transition holds for $\mathrm{MnO}$, which has also $\mathrm{NaCl}$ structure and exhibits the same type of antiferromagnetic order [23]. However, $\mathrm{MnO}$ undergoes a clearly visible discontinuous tetragonal lattice distortion at $T_{N}$ and also the susceptibility reveals a discontinuous behaviour [23]. These features are absent in the case of EuTe or at least they are so small that they could as yet not be verified experimentally. Attempts to observe a spontaneous magnetostriction failed $[20,24]$ and also the susceptibility does not indicate any discontinuity. In the case of $\mathrm{CoO}$, on the other hand, the tetragonal lattice distortion is continuous [25] and positive $\alpha$ values close to the $3 d$ Ising prediction are observed in the magnetic specific heat [26]. This result has been explained with the magnetic anisotropy of $\mathrm{CoO}$. EuTe has certainly a much smaller magnetic anisotropy $[1,15]$ and nevertheless a much larger $\alpha$ value. Hence we conclude that for $B_{\mathrm{ex}}^{q} / B_{\mathrm{ex}}^{1}>0 \mathrm{Eu}_{x} \mathrm{Sr}_{1-x} \mathrm{Te}$ approaches a first-order transition but this situation is not yet reached for EuTe. Interestingly, a phase transition of first order has been predicted by Nagaev and Kovalenko $[27,28]$ if a biquadratic exchange term is added to the 
common bilinear exchange term. Then an "order-alien disorder" transition of first order is possible and a positive $\Theta$ value may be observed even for an antiferromagnet. In fact, the biquadratic interaction has a second energetic minimum for a ferromagnetic spin alignment (Fig. 5) and this minimum will be populated in the paramagnetic state, resulting in ferromagnetic spin correlations which, as a consequence, shift the paramagnetic ordering temperature towards positive values. We expect that this aspect will come out more clearly if EuTe is subjected to external pressure. Since $\mathrm{SrTe}$ has a larger lattice constant (Fig. 1), pressure acts like increasing $x$. The available literature data [29] confirm that the susceptibility decreases stronger under pressure at $T_{N}$.

\section{Conclusions}

As has been known for some time, the low-temperature magnetization curve of EuTe increases faster than linearly with applied field $[1,7,8]$. Our investigations of SrTediluted EuTe samples provided evidence for the main cause of this peculiar behaviour to be an additional biquadratic interaction term rather than field dependent zero-point spin fluctuations [7]. The observed effects are too large to be explained by this mechanism [12] and change too strongly with composition. In particular, zeropoint fluctuations would not allow for a change in sign of the observed effect at $x \approx 0.83$ since the spin order remains essentially unchanged, compare however [40]. An analysis of the nonlinearity of the low-temperature magnetization curves allows a separate evaluation of the bilinear and biquadratic contributions to the molecular field because these add differently for a parallel or antiparallel orientation of both sublattice magnetizations. For $\mathrm{EuTe}$, for instance, the molecular field is $8.5 \mathrm{~T}$ in the antiferromagnetic ground state but $7.5 \mathrm{~T}$ in the ferromagnetically aligned state and this decreasing interaction strength is the reason why the magnetization increases stronger than linearly with applied field. With the interaction parameters obtained in this way, we were able to explain the slope of the Néel line $T_{N}(x)$ which is 1.36 if normalized to $T_{N}(x=1)$, as well as the slope of the critical field $B_{c}(x)$, which is only 0.62 normalized to $x=1$. We consider this twofold agreement as a justification of our analysis.

One further surprising result is the rise of a phase transition at finite fields between antiferromagnetic and spin-flop state for $x<0.83$. Above this composition continuous rotations of the magnetization into the magnetic easy directions occur on returning the field back to zero. This could be confirmed by the absence of magnetocaloric anomalies. For $x<0.83$, however, the biquadratic interaction favours a perpendicular orientation of both sublattice magnetizations and this acts as a new anisotropy which is sufficiently strong to induce a critical AF-SF phase transition at finite fields. Since all phase boundaries are of second order they cannot meet at one point [16], hence precluding a multicritical behaviour. Instead, the SF phase becomes ground state for a small temperature interval and the genuine antiferromagnetic phase, in which the moment orientations are determined by the anisotropies present in the system, is restricted to smaller temperatures and to field values which compare with the biquadratic molecular field $(0.3 \mathrm{~T})$. The character of the order-disorder transition does not seem to change with $x$, leading always from the paramagnetic into the spin-flop state.

It is surprising that the relatively small biquadratic interaction appears to affect the critical behaviour in $\mathrm{Eu}_{x} \mathrm{Sr}_{1-x} \mathrm{Te}$. It is tempting to attribute the anomalous values of the critical exponents for the magnetic specific heat, $\alpha$, and for the sublattice magnetization, $\beta$, to the biquadratic interaction. For EuTe both quantities deviate from the Heisenberg-model prediction in a sense, suggesting that the phase transition at $T_{N}$ could be weakly of first order as has been noted before [20]. However, there is no definite experimental evidence supporting this assumption. In particular, no discontinuous lattice change at $T_{N}$ could be detected $[20,24]$. On the other hand, in systems like $\mathrm{CoO}$ with an uncompletely quenched orbital moment $[25,26]$, a tetragonal lattice distortion of the Jahn-Teller type develops near $T_{N}$, lifting the existing degeneracy [30] and as a consequence of this reduced symmetry the asymptotic critical behaviour changes from Heisenberg like to Ising like and a positive $\alpha$ value of $\alpha=+1 / 8$ in agreement with the Ising prediction is in fact observed [26]. In EuTe, however, such an anisotropy is very much smaller, if it exists at all. The $\mathrm{Eu}^{2+}$ moment can be considered as a pure spin moment but nevertheless the observed asymptotic $\alpha$ value exceeds the Ising prediction by far. We are therefore led to conclude that EuTe is close to a transition of first order which will be driven by an instability due to the biquadratic exchange but the requirements for this event are not completely met.

The introduction of a biquadratic type of interaction in $\mathrm{Eu}_{x} \mathrm{Sr}_{1-x} \mathrm{Te}$ in the present work was purely on phenomenological grounds. The existence of this interaction could be verified in a number of other systems too [31-33] and has been treated theoretically [34]. For EuSe a nearest-neighbour biquadratic exchange parameter has been evaluated from an analysis of the anisotropy of lowtemperature magnetization curves [6]. The observed sign agrees with that one found here for EuTe. Calculating the total exchange energy to the twelve nearest neighbours using this parameter one obtains $1.55 \mathrm{~K}$ for EuSe. This number should be compared with $7 \mu_{B} \cdot B_{\mathrm{ex}}^{q}$ which corresponds to $2.35 \mathrm{~K}$ if $B_{\mathrm{ex}}^{q}=0.5 \mathrm{~T}$, obtained for EuTe is inserted. It seems therefore that the importance of the biquadratic interaction increases towards EuTe in the $\mathrm{Eu}-$ monochalcogenide series, if we bear in mind that the strength of this interaction is below the experimental limits in EuS and EuO, as can be concluded from the critical behaviour of these two materials, which is in good agreement with the $3 d$ Heisenberg expectation $[18,21,22]$. Further work has to be done towards a complete understanding of this peculiar magnetic interaction whose microscopic origin is yet unclear.

We thank P. Ehrhart for performing the lattice constant measurements. Critical comments on the manuscript received by $\mathrm{K}$. Binder and $\mathrm{W}$. Selke are acknowledged with gratitude. Thanks are also to C. Juliusson for performing the MCE measurements. The technical assistance of F. Deloie is also very much appreciated. 


\section{References}

1. Oliveira Jr., N.F., Foner, S., Shapira, Y., Reed, T.B.: Phys. Rev. B5, 2634 (1972)

2. Will, G., Pickart, S.J., Alperin, H.A., Nathans, R.: J. Phys. Chem. Solids 24, 1679 (1963)

3. Streit, P.K., Everett, G.E.: Phys. Rev. B21, 169 (1980)

4. Bohn, H.G., Zinn, W., Dorner, B., Kollmar, A.: Phys. Rev. B22, 5447 (1980)

5. Zinn, W.: J. Magn. Magn. Mater. 3, 23 (1976)

6. Fukuma, H., Komatsubara, T., Suzuki, T., Kaldis, E., Kasuya, T.: J. Phys. Soc. Jpn. 54, 3067 (1985)

7. Jacobs, I.S., Silverstein, S.D.: Phys. Rev. Lett. 13, 272 (1964)

8. Schwarz, B.R.I., Lütgemeier, H., Zinn, W.: J. Magn. Magn. Mater. 45, 369 (1984)

9. Börgermann, F.-J., Maletta, H., Zinn, W.: Phys. Rev. B35, 8454 (1987)

10. Scheer, E., Wosnitza, J., Löhneysen, H. v.: Z. Phys. B85, 79 (1991)

11. Nowotny, R.M., Binder, K.: Z. Phys. B77, 287 (1989)

12. Swendsen, R.H.: J. Phys. C6, 3763 (1973)

13. Köbler, U., Binder, K.: J. Magn. Magn. Mater. 15-18, 313 (1980)

14. Stinchcombe, R.B.: In: Phase transitions and critical phenomena. Domb, C., Lebowitz, J.L. (eds.), Vol. 7, p. 151. New York: Academic Press 1983

15. Battles, J.W., Everett, G.E.: Phys. Rev. B1, 3021 (1970)

16. Yip, S.K., Li, T., Kumar, P.: Phys. Rev. B43, 2742 (1991)
17. Shirane, G.: Acta Crystallogr. 12, 282 (1959)

18. Menyuk, N., Dwight, K., Reed, T.B.: Phys. Rev. B3, 1689 (1971)

19. Le Guillon, J.C., Zinn-Justin, J.: Phys. Rev, B21, 3976 (1980)

20. Scheer, E., Wosnitza, J., Löhneysen, H. v., Kürsch, R., Lang, M., Steglich, F.: J. Magn. Magn. Mater. 104-107, 175 (1992)

21. Wosnitza, J., Löhneysen, H. v.: Europhys. Lett. 10, 381 (1989)

22. Kornblit, A., Ahlers, G., Buehler, E.: Phys. Rev. B17, 282 (1978)

23. Jagadeesh, M.S., Seehra, M.S.: Phys. Rev. B23, 1185 (1981)

24. Levy, F.: Phys. Kondens. Mater. 10, 71 (1969)

25. Silinsky, P.S., Seehra, M.S.: Phys. Rev, B24, 419 (1981)

26. Salamon, M.B.: Phys. Rev. B2, 214 (1970)

27. Nagaev, E.L., Kovalenko, A.A.: Solid State Commun. 38, 1121 (1981)

28. Nagaev, E.L., Kovalenko, A.A.: Sov. Phys.-JETP 52, 460 (1980)

29. Schwob, P.: Phys. Kondens. Mater. 10, 186 (1969)

30. Rechtin, M.D., Averbach, B.L.: Phys. Rev. Lett. 26, 1483 (1971)

31. Sakakibara, T., Date, M.: J. Phys. Soc. Jpn. 53, 3599 (1984)

32. Harris, E.A., Owen, J.: Phys. Rev. Lett. 11, 9 (1963)

33. Rodbell, D.S., Jacobs, I.S., Owen, J.: Phys. Rev. Lett. 11, 10 (1963)

34. Herring, C.: Magnetism IIB. Rado, G.T., Suhl, H. (eds.). New York: Academic Press 1966

35. Harris, A.B.: J. Phys. C7, 1671 (1974)

36. Shuil, C.G., Strauser, W.A., Wollan, E.O.: Phys. Rev. 83, 333 (1951)

37. Li, Y.-Y.: Phys. Rev. 100, 627 (1955)

38. Keffer, F., O'Sullivan, W.: Phys. Rev. 108, 637 (1957)

39. Roth, W.L.: Phys. Rev. 111, 772 (1958)

40. Henley, C.L.: J. Appl. Phys. 61, 3962 (1987) 